\title{
OCCUPANCY MODELLING FOR MOVING OBJECT DETECTION FROM LIDAR POINT CLOUDS: A COMPARATIVE STUDY
}

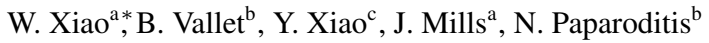 \\ ${ }^{a}$ School of Engineering, NEOlab, Newcastle University, Newcastle upon Tyne, UK - wen.xiao@ newcastle.ac.uk \\ ${ }^{\mathrm{b}}$ Université Paris-Est, LaSTIG MATIS, IGN, ENSG, Saint-Mandé, France \\ ${ }^{\mathrm{c}}$ Department of Civil and Environmental Engineering, University of Michigan, Ann Arbor, USA
}

Commission II, WG II/3

KEY WORDS: Laser Scanning, Change Detection, Dynamic Scene, Occupancy Grid, Probability, Dempster-Shafer Theory

\begin{abstract}
:
Lidar technology has been widely used in both robotics and geomatics for environment perception and mapping. Moving object detection is important in both fields as it is a fundamental step for collision avoidance, static background extraction, moving pattern analysis, etc. A simple method involves checking directly the distance between nearest points from the compared datasets. However, large distances may be obtained when two datasets have different coverages. The use of occupancy grids is a popular approach to overcome this problem. There are two common theories employed to model occupancy and to interpret the measurements, DempsterShafer theory and probability. This paper presents a comparative study of these two theories for occupancy modelling with the aim of moving object detection from lidar point clouds. Occupancy is modelled using both approaches and their implementations are explained and compared in details. Two lidar datasets are tested to illustrate the moving object detection results.
\end{abstract}

\section{INTRODUCTION}

Laser scanning, also referred to as lidar (light detection and ranging), has been used in both robotics and geomatics for slightly different purposes. In robotics, it is initially used for environment perception to avoid collision. Nowadays, it is widely used for autonomous navigation and SLAM (simultaneous localization and mapping) (Wolf and Sukhatme, 2004; Moosmann and Stiller, 2013), so that a robot will know exactly where it is, even in a new environment. In geomatics, mapping is usually the primary goal, and laser scanning can provide a precise way of mapping the environment (Aijazi et al., 2013; Zhou and Vosselman, 2012). There are specific laser scanners for precise and dense mapping, for both indoor and outdoor, that can be operated from different platforms such as aircraft, mobile vehicles, and terrestrial tripods. One type of laser scanner that has been adopted in both robotics and geomatics studies is the Velodyne HDL-64E, which consists of 64 laser rays rotating rapidly around the vertical axis so that the surroundings are continuously scanned. Continuous scanning is essential in robotics as a robot needs to see the environment all the time. This type of real-time laser scanner has been widely used for autonomous driving and environment mapping (Moosmann and Stiller, 2011; Hornung et al., 2013).

Moving object detection and tracking is an important research topic for both robotics and geomatics (Lindström and Eklundh, 2001; Kaestner et al., 2012; Xiao et al., 2016). For the former, a robot, such as an autonomous vehicle needs to not only see its surroundings, but also make sure its trajectory will not coincide with other moving objects in a dynamic environment. Therefore, it is necessary to track and even predict other moving objects to avoid any potential collision. As for the latter, the static environment is of primary interest for mapping purposes. So moving object detection and removal will help clean the environment and reduce the data storage (Vallet et al., 2015). There are often movable

${ }^{*}$ Corresponding author objects that remain static in the data during the process of data acquisition, such as parked cars along the street. In such cases, detected moving objects can serve as training samples for comprehensive and fully automatic mobile object removal. Moreover, object moving patterns, such as pedestrian trajectories in a public space or vehicle movement in a congregated crossroads, are potentially valuable geoinformation for further studies.

There are various methods for moving object detection from both robotics and geomatics. For example, a straightforward method is calculating the point to point distance between two different point clouds (Girardeau-Montaut et al., 2005; Xu et al., 2013; Lindenbergh and Pietrzyk, 2015), which can be two different scans. As for a real-time laser scanner, a scan refers to a full $360^{\circ}$ rotation of the scanner. Apart from simple point to point distance, there exist improved variants such as computing the distance between a point and a local surface, either a plane or a triangle generated from local neighbour points. This method is simple and fast and still used in many applications. However, it is not able to distinguish points on moving objects from points that have no correspondence, which happens when two scans do not cover the same space, such as in occlusions. In both cases, these point will have large distances indicating movements. Another method, occupancy grids, has been used initially in robotics for environment perception (Collins, 2011; Vu et al., 2011; Thrun, 2002), and recently in geomatics for change detection (Hebel et al., 2013; Xiao et al., 2015). It models the occupancy information of the space, i.e. whether a specific location (often a grid cell in 2D) is occupied by an object or not. This information is derived from the measured environment data. The occupancy states will vary by time in a dynamic environment. One of the advantages is that it is able to capture cells that have no correspondence, as all cells will be initiated but those will not be compared or updated.

To detect the changes of occupancy states so as to detect moving objects, the occupancy need to be defined or modelled first. One approach is using probability to represent the occupancy of space. 
Then Bayes' rule is used to interpret measurements and update the probability (Thrun, 2002; Collins, 2011; Vu et al., 2011; Hornung et al., 2013). Another common approach is the DemspterShafer theory (DST) (Shafer, 1992), an evidence theory that combines evidence from different sources and reaches a certain degree of belief (Murphy, 1998; Grabe et al., 2009; Delafosse et al., 2007; Moras et al., 2015; Dezert et al., 2015). There are other occupancy modelling methods such as fuzzy logic (Payeur, 2003), but the former two are mostly widely used for occupancy modelling and moving object detection or change detection. This paper will investigate in detail the two methods in terms of both theory and implementation. This comparison will help the users to select the appropriate approach for their specific applications.

\section{METHOD}

In this section the occupancy of a laser scan is modelled using both Dempster-Shafer theory (DST) and probabilistic model approaches. The occupancy definition is introduced first, then their occupancy information is fused, followed by the identification of the occupancy consistency between multiple scans.

\subsection{Occupancy Definition}

2.1.1 Occupancy by Dempster-Shafer theory Theoretically, the space can be either occupied or free, which are the two occupancy states. Based on DST, the occupancy is defined by the universal set $X=\{$ free, occupied $\}$. And the power set of $X$, $2^{X}=\{\emptyset,\{$ free $\},\{$ occupied $\},\{$ free, occupied $\}\}$, which contains all the subsets of $X$. In practise, if an area is not measured, there is zero knowledge about the occupancy state, meaning the occupancy is unknown. Then the space can be either free or occupied, so the subset $\{$ free, occupied $\}$ in the power set represents the unknown situation. The DST is defined as follows:

$$
M: 2^{X} \rightarrow[0,1], M(\emptyset)=0, \sum_{A \in 2^{X}} M(A)=1
$$

in which $M(A)$ is the occupancy function, including the three occupancy states, namely free $M(f)$, occupied $M(o)$ and unknown $M(u)$. They are in the range of $[0,1]$, and their sum equals to 1 .

The occupancy needs to be modelled in 3D for the 3D laser scanning measurements. Along the direction of a laser ray $r$, the occupancy is modelled in the following way. The laser ray travels through the space and is then reflected back from an object where a point is defined. So it is known to be free between the laser scanner center and the point, $f_{r}=1 ; o_{r}=0$. At the location of the point, an object is supposed to be present, thus it is certainly occupied, $f_{r}=0 ; o_{r}=1$. Behind the point, or ahead of the point along the ray, the space is not measured so its occupancy state is unknown $u_{r}=1$. As an object will definitely be larger than a single point, the space behind the point can be assumed to be occupied within a certain range. Beyond that range, the occupancy will become completely unknown. The occupancy modelling is illustrated in Figure 1.

Xiao et al. (2015) modelled the maximum occupancy slightly behind the point as it was argued that the point is on the surface of an object, then considering uncertainties, it can be on either side of the surface. Thus the occupancy is actually half free and half occupied at this very location. By convolving with an uncertainty Gaussian function, the maximum of occupied is slightly behind

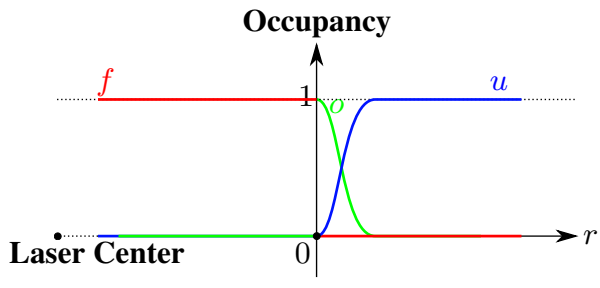

Figure 1. Occupancy in ray direction

the point, which is true since the space is more certain to be occupied behind the surface. In this way, the occupancy along the ray is accurately modelled. However, the occupancy consistency assessment is then complicated as this will end up with half consistent and half conflicting for two points at the same location (see Section 2.3.1). So the maximum occupancy at the location of the point is modelled as shown in Figure 2.

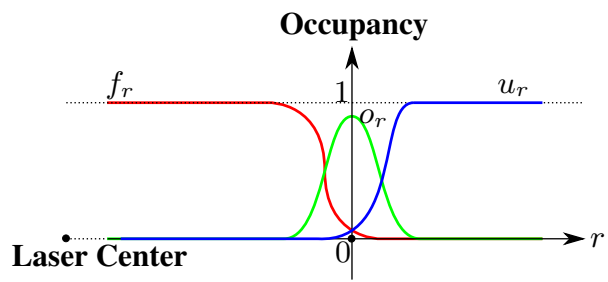

Figure 2. Occupancy in ray direction considering uncertainties

Now, the occupancy has been defined only in the ray direction. In $3 \mathrm{D}$, the occupancy around a laser ray can be extrapolated to a certain extent based on the distance to the ray itself. To facilitate the extrapolation, the distance to a laser ray can be computed in the laser scanner's own local coordinate system. Here, it is composed of the vertical angular direction $\theta$, trajectory direction $t$, and the ray direction $r$. The occupancy is at a maximum along the ray, and it decreases along the other two directions. The 3D occupancy modelling is depicted in Figure 3.

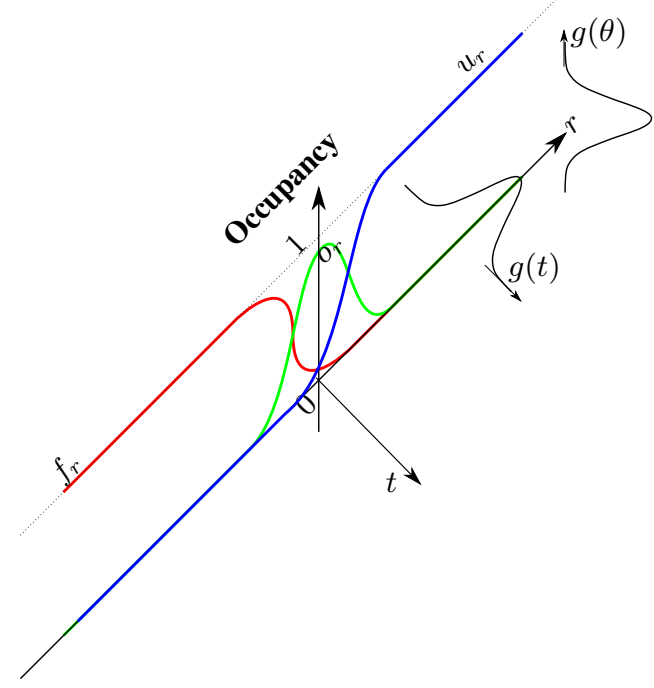

Figure 3. Occupancy in 3D considering uncertainties

The occupancy in rotation $\theta$ and trajectory $t$ directions is extrapolated using Gaussian functions $g_{\theta}$ and $g_{t}$. The standard deviations are determined by the gaps between rays in respective directions. In practise, sampling densities are not the same in these two directions. To overcome this anisotropic sampling issue, the occupancy in these two directions are interpolated separately. The 
overall occupancy of a point is a function of the three variables, $r, t, \theta$ :

$$
M(A)=M\left\{\begin{array}{l}
f \\
o \\
u
\end{array}\right\}=\left\{\begin{array}{l}
g_{\theta} \cdot g_{t} \cdot f_{r} \\
g_{\theta} \cdot g_{t} \cdot o_{r} \\
1-f-o
\end{array}\right\}
$$

in which $g_{\theta}, g_{t} \in[0,1]$ are interpolation coefficients.

2.1.2 Probabilistic Occupancy The principle of probabilistic occupancy is similar to DST, where space is free along the laser beam and occupied at the location of the laser point. The probability $P \in[0,1]$ represents the status of occupancy, i.e. $P=1$ when the space is occupied, $P=0$ when it is free. Figure 4 shows the definition of probabilistic occupancy.

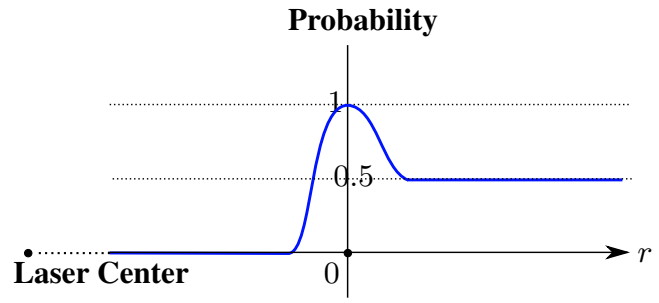

Figure 4. Probabilistic occupancy in ray direction

From the laser scanner center to the point, the laser beam has travelled through the space that is free, hence the probability is 0 . At the location of the point, the space is occupied. Due to laser range measurement errors and positioning errors from the mapping system, the point position has certain degree of uncertainty, which can be accounted for by coupling a random error distribution to the probability both behind and ahead of the point along the ray direction. Further ahead of the point along the ray, the space is not scanned hence can be either free or occupied. Then the probability of occupancy is defined as 0.5 . Different from DST, the probabilistic occupancy only has one value to represent the space's occupancy status.

\subsection{Occupancy Fusion}

The space will be scanned multiple times by the laser scanner, meaning there will be multiple measurements at the same location. The probabilities of different scans need to be fused for final inference. This section will discuss and compare the two occupancy fusion methods.

2.2.1 Dempster's Rule of Combination After defining the occupancy function $M(A)$ based on DST, the occupancy of different rays can be fused by the Dempster's rule of combination which is as follows:

$$
\left(M_{1} \oplus M_{2}\right)(A)=\frac{1}{1-K} \sum_{B \cap C=A \neq \emptyset}\left(M_{1}(B) \cdot M_{2}(C)\right)
$$

in which $B \in(f, o, u), C \in(f, o, u)$ and $K$ is the conflict between these two sets $K=\sum_{B \cap C=\emptyset}\left(M_{1}(B) \cdot M_{2}(C)\right)$.

Note that $\{f\}$ and $\{o\}$ are the subsets of $\{u\}$. So the combined occupancy of two rays $M_{1} \oplus M_{2}$ is:

$$
\left\{\begin{array}{l}
f_{1} \\
o_{1} \\
u_{1}
\end{array}\right\} \oplus\left\{\begin{array}{l}
f_{2} \\
o_{2} \\
u_{2}
\end{array}\right\}=\frac{1}{1-K}\left\{\begin{array}{l}
f_{1} \cdot f_{2}+f_{1} \cdot u_{2}+u_{1} \cdot f_{2} \\
o_{1} \cdot o_{2}+o_{1} \cdot u_{2}+u_{1} \cdot o_{2} \\
u_{1} \cdot u_{2}
\end{array}\right\}
$$

in which $K=o_{1} \cdot f_{2}+f_{1} \cdot o_{2}$.

One important character of Dempster's combination rule is that it is commutative and associative, meaning the occupancy from different rays can be combined in any order. For this reason other combination rules are not chosen, such as Yager's rule which is not associative (Yager and Liu, 2008). Given $I$ number of neighbouring rays $R_{i}$, the overall occupancy is:

$$
M(A)=\bigoplus_{i \in I} M\left(A, R_{i}\right)
$$

2.2.2 Probability Interpretation and Log-odd Simplification Similarly, a location in the space can be scanned multiple times, then the probability of this location has to be updated. So the objective is to estimate the posterior probability of occupancy $P\left(m \mid z_{1: t}\right)$ of each location $m$ given the measurements $z_{1: t}=$ $\left\{z_{1}, \ldots, z_{t}\right\}$ (Hornung et al., 2013).

$$
\begin{aligned}
P\left(m \mid z_{1: t}\right) & =\frac{P\left(z_{t} \mid m\right) P\left(m \mid z_{1: t-1}\right)}{P\left(z_{t} \mid z_{1: t}\right)} \\
& =\frac{P\left(m \mid z_{t}\right) P\left(z_{t}\right) P\left(m \mid z_{1: t-1}\right)}{P(m) P\left(z_{t} \mid z_{1: t}\right)}
\end{aligned}
$$

This gives the probability of being occupied, then the probability of being free $P(\bar{m})=1-P(m)$. Then,

$$
P\left(\bar{m} \mid z_{1: t}\right)=\frac{P\left(\bar{m} \mid z_{t}\right) P\left(z_{t}\right) P\left(\bar{m} \mid z_{1: t-1}\right)}{P(\bar{m}) P\left(z_{t} \mid z_{1: t}\right)}
$$

By dividing Equations 6 and 7, some terms are eliminated (Thrun, 2002).

$$
\frac{P\left(m \mid z_{1: t}\right)}{P\left(\bar{m} \mid z_{1: t}\right)}=\frac{P\left(m \mid z_{t}\right)}{P\left(\bar{m} \mid z_{t}\right)} \frac{P(\bar{m})}{P(m)} \frac{P\left(m \mid z_{1: t-1}\right)}{P\left(\bar{m} \mid z_{1: t-1}\right)}
$$

Given a probability $P(m)$, the odds is defined as odds $(m)=$ $P(m) /(1-P(m))$, and the logarithm log-odds is $L(m)=$ $\log \left(\frac{P(m)}{1-P(m)}\right)$. Then Equation 8 can be rewritten as:

$$
L\left(m \mid z_{1: t}\right)=L\left(m \mid z_{t}\right)-L(m)+L\left(m \mid z_{1: t-1}\right)
$$

Here, $P(m)$ is the prior occupancy probability which is set to 0.5 representing an unknown state, then $L(m)=0$, so

$$
L\left(m \mid z_{1: t}\right)=L\left(m \mid z_{t}\right)+L\left(m \mid z_{1: t-1}\right)
$$

The probability updating is transformed to a simple addition of the previous log-odds and the log-odds of the current measurement. The logit function of probability is illustrated in Figure 5. 
Probability $P \in[0,1]$ and $\log$-odds $\in[-\infty,+\infty]$. Note that the occupancy probability can easily be transferred to a log-odds value, and vice versa. Due to uncertainty, $P$ will hardly reach 1 , and hence its log-odds will not be $\infty$. In practice, log-odds values, $l_{\text {occ }}$ and $l_{\text {free }}$ are defined to represents the occupied and free states of a single measurement. So Figure 4 is transformed to log-odds domain as shown in Figure 6.

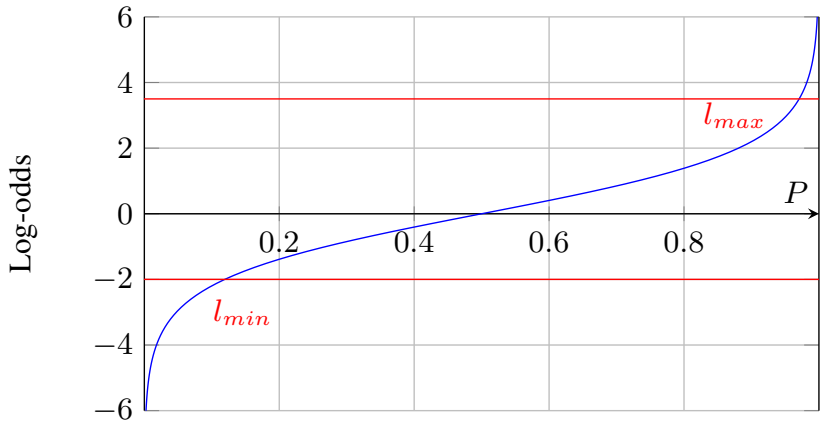

Figure 5. Log-odds of probability in the domain of -6 to 6

Given a laser beam, the occupancy is $l_{\text {free }}$ from the laser scanner center and increases to $l_{o c c}$ at the location of the point. To simplify the log-odds function for a laser beam, the transition from free to occupied is set to be linear, as also the case for the transition to unknown, where $l_{o c c}$ is decreased to 0 (Figure 6).

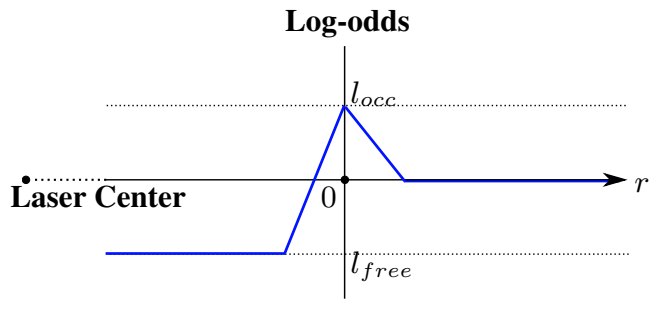

Figure 6. Log-odds in ray direction

For multiple measurements at the same location, their log-odds values are simply summed. Based on the definition, it can be observed that to change an occupancy state, it needs at least the same number of observations that define its current state. It means that if a location is consolidated $n$ times by laser beams as free, another $n$ times of occupied measurement will be needed before identifying the location as being occupied. In a dynamic environment, an object will move into a free space and leave immediately after, depending on its speed of movement. To capture this kind of rapid change, a clamping threshold is defined to update the occupancy states (Yguel et al., 2007). Once the log-odds value reaches a threshold, either lower bound $l_{\min }$ or $l_{\max }$, the space occupancy state will no longer be updated. So $l_{\min }$ or $l_{\max }$ are thresholds where the space is inferred as free or occupied (red in Figure 5).

2.2.3 Comparison between Dempster's Rule of Combination and Bayes' Rule Defined by DST, space occupancy $M(A)$ can have three states, free $M(f)$, occupied $M(o)$, and unknown $M(u)$, and the sum $\sum M(A)=1$. By the probabilistic model, the space only has two occupancy states, either free $P(f)$ or occupied $P(o)$, and their sum also equals 1 , meaning $P(f)=P(\neg o)=1-P(o)$. For where the occupancy is unknown, the space is defined as half-free and half-occupied, $P(f)=P(o)=0.5$.

To fuse multiple measurements, Dempster's rule of combination is as given in Equation 3. Bayes' rule for an event of combined probability is as follows:

$$
P(A \mid B)=\frac{P(B \mid A) P(A)}{P(B \mid A) P(A)+P(B \mid \neg A) P(\neg A)}
$$

In the case of probabilistic occupancy, the combined probability of two rays will be

$$
P\left(o_{12}\right)=\frac{P\left(o_{1}\right) P\left(o_{2}\right)}{P\left(o_{1}\right) P\left(o_{2}\right)+P\left(e_{1}\right) P\left(e_{2}\right)}
$$

For DST, according to Equation 3, the mass of occupied is

$$
M\left(o_{12}\right)=\frac{M\left(o_{1}\right) M\left(o_{2}\right)+M\left(o_{1}\right) M\left(u_{2}\right)+M\left(u_{1}\right) M\left(o_{2}\right)}{1-M\left(o_{1}\right) M\left(e_{2}\right)-M\left(e_{1}\right) M\left(o_{2}\right)}
$$

In case of $M(u)=0$, the equation will become

$$
M\left(o_{12}\right)=\frac{M\left(o_{1}\right) M\left(o_{2}\right)}{M\left(o_{1}\right) M\left(o_{2}\right)+M\left(e_{1}\right) M\left(e_{2}\right)}
$$

which is essentially the same as Equation 12. So, for locations where $M(u)=0$, meaning whenever there is information of occupancy, the two theories, DST and Bayes' rule, give the same result. The space between the laser scanner center and the point, in other words, the space scanned by the laser should have similar occupancy results from these two theories. However, due to uncertainties, $M(u)$ will hardly be exactly 0 . Therefore, in reality, some differences are expected. Behind the point when $M(o)=M(u)=0.5$, given two similar laser rays, the combined occupancy $M\left(o_{12}\right)=0.75$, whereas $P\left(o_{12}\right)=0.5$. So the occupancy evidence can still be accumulated behind the point. The DST is able to distinguish half free half occupied and half occupied half unknown, whereas the Bayes's rule cannot.

\subsection{Consistency Assessment}

After combining occupancy from different rays within one scan, occupancies from different scans can be compared. This section assesses the consistency between scans to find out points on moving objects.

As occupancy is often modelled in 3D voxels (Hebel et al., 2013), the consistency can be easily assessed on each voxel. However, the voxelization of the 3D space can be computationally expensive. A better approach is to perform the assessment directly on each individual points without voxelization. Point-level consistency assessment is performed for each of the occupancy modelling method.

2.3.1 Dempster-based Consistency The occupancy consistency is defined as conflicting when one scan is empty and the other scan is occupied or vice versa, consistent when they have the same occupancy state, and uncertain if one dataset is unknown whereas the other one is known. The consistency relations between two scans, $\left(F_{1}, O_{1}, U_{1}\right)$ and $\left(F_{2}, O_{2}, U_{2}\right)$, are defined as:

$$
\begin{aligned}
& \text { Conflict }=F_{1} \cdot O_{2}+O_{1} \cdot F_{2} \\
& \text { Consist }=F_{1} \cdot F_{2}+O_{1} \cdot O_{2}+U_{1} \cdot U_{2} \\
& \text { Uncertain }=U_{1} \cdot\left(F_{2}+O_{2}\right)+U_{2} \cdot\left(F_{1}+O_{1}\right)
\end{aligned}
$$


The occupancy of a point is known $M(A)=\left(f_{1}, o_{1}, u_{1}\right) \simeq$ $(0,1,0)$ (Figure 2). Then to find out whether this point is on an moving object, one only needs to compute the occupancy of the compared scans at the location of this point. Then the consistency equations will be simplified as:

$$
\begin{array}{ll}
\text { Conflict } & =f_{1} \cdot O_{2}+o_{1} \cdot F_{2} \simeq F_{2} \\
\text { Consist } & =f_{1} \cdot F_{2}+o_{1} \cdot O_{2}+u_{1} \cdot U_{2} \simeq O_{2} \\
\text { Uncertain } & =u_{1} \cdot\left(F_{2}+O_{2}\right)+U_{2} \cdot\left(f_{1}+o_{1}\right) \simeq U_{2}
\end{array}
$$

So, if the occupancy at the location of a point is free, it is conflicting with the point, and the point should have moved in the space, meaning it is on a moving object. If the occupancy is occupied, the point has not moved. And if it is unkown, the point lies behind or out of the scope of the compared rays, such as in the shadow, then it is uncertain whether the point is moving or not.

\subsubsection{Probability-based Consistency During laser scanning,} each scan and each beam is considered as independent. Also, neighbouring beams are assumed to be independent. Moving object detection amounts to checking whether the occupancy of a location is consistent in which case the environment is static. Similar to the DST consistency assessment, only the points themselves have to be identified as moving or not, which means we only need to check the consistency of each point instead of the entire space. The space can be free or occupied, so consistency means two scans both report the space as either free or occupied. Similarly, inconsistency means two scans give contrary evidence.

$$
\begin{aligned}
& P\left(\left(o_{1} \cup o_{2}\right) \cap\left(e_{1} \cup e_{2}\right)\right)=P\left(o_{1}\right) P\left(o_{2}\right)+P\left(e_{1}\right) P\left(e_{2}\right) \\
& P\left(\left(o_{1} \cup e_{2}\right) \cap\left(e_{1} \cup o_{2}\right)\right)=P\left(o_{1}\right) P\left(e_{2}\right)+P\left(e_{1}\right) P\left(o_{2}\right)
\end{aligned}
$$

A point's occupancy probability $P(o)=1$, and $P(e)=0$. Then the consistency will simply be

$$
\begin{aligned}
& P\left(\left(o_{1} \cup o_{2}\right) \cap\left(e_{1} \cup e_{2}\right)\right)=P\left(o_{2}\right) \\
& P\left(\left(o_{1} \cup e_{2}\right) \cap\left(e_{1} \cup o_{2}\right)\right)=P\left(e_{2}\right)
\end{aligned}
$$

Therefore, to find out whether a point is on a moving object, we only need to check the occupancy of the validating rays that have influence at the location of the point. If the occupancy state is occupied, meaning the space is consistent and the point is static. Otherwise, the point is on a moving object.

\section{MOVING OBJECT DETECTION}

Two datasets acquired by a mobile laser scanning system are selected for moving object detection. In the first data, the scanning system is static, aiming at constantly scanning a public space for moving object detection and tracking. In the second, the system is moving whilst scanning the street for the purpose of urban mapping and modelling. The experimental data were acquired in Paris using the Stereopolis mobile mapping system, which is georeferenced by the combination of GNSS and IMU, with a HDL$64 \mathrm{E}$ Velodyne laser scanner at a frequency of $10 \mathrm{~Hz}$ (Paparoditis et al., 2012). The detection results are quantitatively evaluated against manually labelled ground truth, which may have a few mislabelled points. But all detections are compared with the same ground truth, so the results are conclusive. Since the method detects points on moving objects, the results are evaluated at point level. Recall (R), precision (P) and $F_{1}$ score are assessed.

\subsection{Static Laser Scanning}

As discussed in Section 2.2.2, the algorithm needs to be sensitive to contradictory measurements to capture quick moving objects. Especially for static laser scanning, the laser will constantly scan the same locations, hence the evidence will quickly become saturated and it will take at least the same amount of opposite evidence to change the occupancy states. For some penetrable objects, e.g. pole-like barriers or trees, laser rays can pass through them and reach the space behind. There will also be some points reflected from such objects, in which case the points on the objects will be conflicting with points behind. This kind of self conflicting will cause false detections on such objects. For real moving objects, there will be little consistent evidence and most of the measurements will give conflicting evidence. So a favour can be given to consistent measurements to reduce false detections. A high weight will be given to consistent evidence if contradictory measurements occur.

In the case of using log-odds for moving object detection, to keep the sensitivity of state changes, especially the sensitivity to consistent evidence, a higher value of $l_{o c c}$ is needed. Same as Hornung et al. (2013), the clamping thresholds $l_{\min }$ and $l_{\max }$ are set to -2 and 3.5 respectively, corresponding to probabilities of 0.12 and 0.97 . If the log-odds summing up value is lower than $l_{\min }$ or higher than $l_{\text {max }}$, the space is determined as free or occupied respectively. The occupancy values $l_{\text {free }}$ and $l_{\text {occ }}$ are set to be -0.4 and 3.0 respectively based on our experiments. Clearly a higher value is assigned to $l_{\text {occ }}$ to reduce false detections. Moving object detection results using both DST and probability theory from a static laser scanner are illustrated in Figure 7.

For moving object detection from static laser scanning, the two occupancy-based methods in this paper are compared to other methods proposed by Xiao et al. (2016), Max-Distance and Nearest-point. The basic principle of the Max-Distance method is straightforward. The static environment is assumed to be impenetrable and only the furthest points of each laser beam are considered to be on static background. Points on moving objects are supposed to be between the laser center and the far end points. The other method, Nearest-point, involves counting the number of nearest points. In the case of static laser scanning, the environment will be continuously scanned. As for static objects, points will be accumulated over time. So these points are assumed to have large number of neighbour points. Whereas for moving objects, they will be scanned at various locations along their trajectories. Due to the high scanning frequency, the moving objects instances are normally overlapped, but still the number of neighbour points will be significantly lower than points on static objects. A temporal window can be defined assuming certain moving speed and a proper object size. The object is supposed to have moved out of its original space within this temporal window, meaning no nearest points are supposed to be found. Note that these two methods are based on simple assumptions and only suitable for static laser scanning scenarios.

Results of the four methods are shown in Table 1. Max-Distance gives the highest recall, as all the points between the laser center and the furthest points are considered as moving points. This is also the reason why the precision is really low. Nearest-point results in the highest precision thanks to the strict thresholding of minimum number of neighbouring points. The two occupancybased methods have better $F_{1}$ scores, meaning their overall performances are better. The probability approach shows a better 


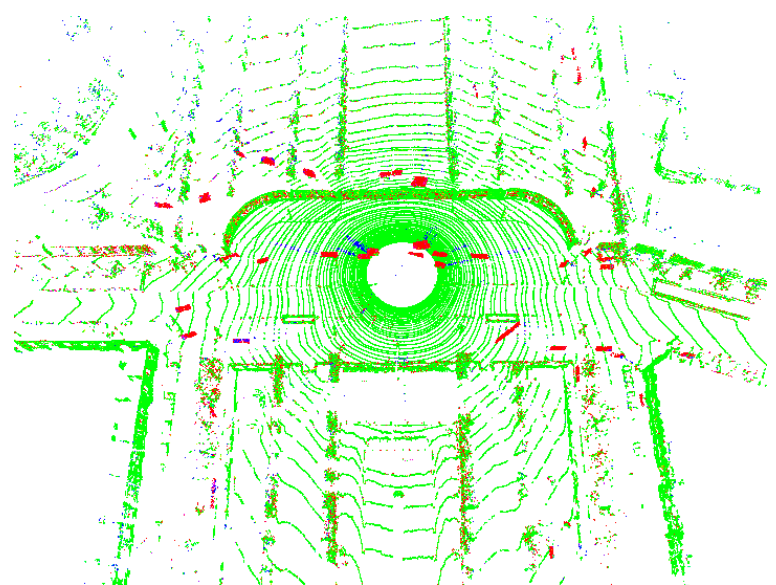

(a)

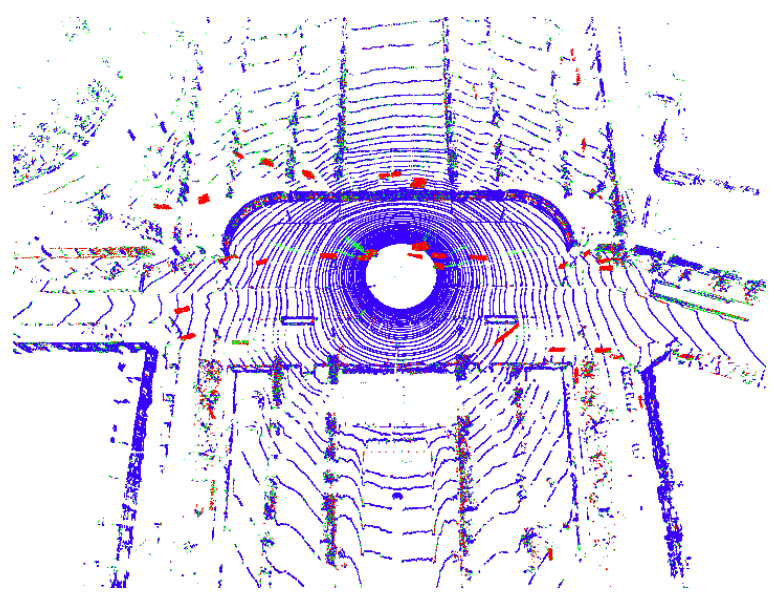

(b)

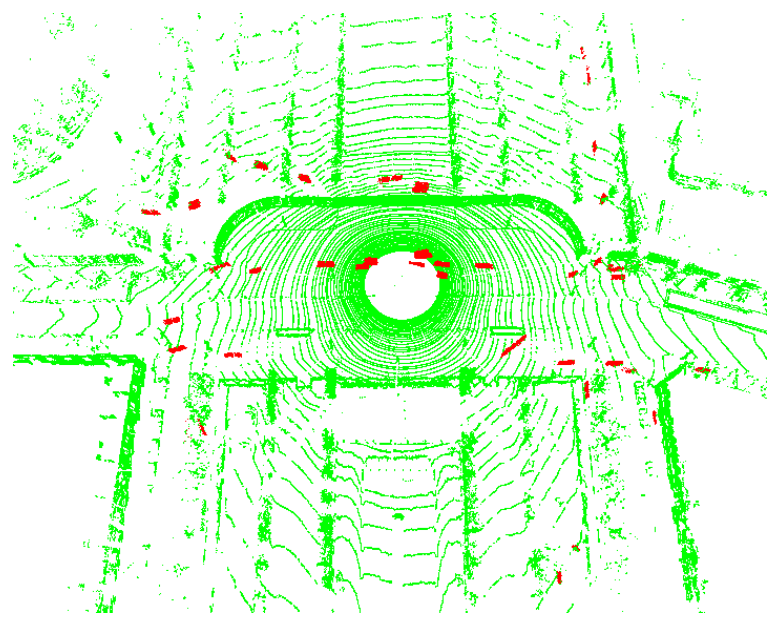

(c)

Figure 7. Moving object detection from a static laser scanner. (a) DST approach (R:moving, G:static, B:uncertain); (b) Probability approach (R:moving, G:uncertain, B:static); (c) ground truth of moving object (R:moving)

balance between recall and precision, and has the highest $F_{1}$ score.

\subsection{Mobile Laser Scanning}

As aforementioned, mobile laser scanning has been widely used for both environment mapping and autonomous navigation. Mov-

\begin{tabular}{|l|l|l|l|}
\hline Method & $\mathrm{R} \%$ & $\mathrm{P} \%$ & $F_{1} \%$ \\
\hline DST & 83.0 & 57.3 & 67.8 \\
Probability & 74.1 & 66.4 & $\mathbf{7 0 . 1}$ \\
Max-Distance & $\mathbf{9 6 . 2}$ & 20.9 & 34.3 \\
Nearest-point & 60.1 & $\mathbf{7 0 . 1}$ & 64.7 \\
\hline
\end{tabular}

Table 1. Accuracy assessment of moving object detection at point level from a static laser scanner.

ing object detection from mobile laser scanning data is a fundamental step in both fields. Occupancy-based methods will manifest their advantages as the laser scanner will cover different areas while moving forward, in which case the method needs to differentiate the real moving points from those that are out of the scanning scope. So the simple methods from the static laser scanning case, Max-Distance and Nearest-point, are no longer applicable. Only the two occupancy-based methods are evaluated. To keep the sensitivity to dynamics in the environment, the same settings as static laser scanning are used. Moving object detection results from a mobile laser scanner are demonstrated in Figure 8.

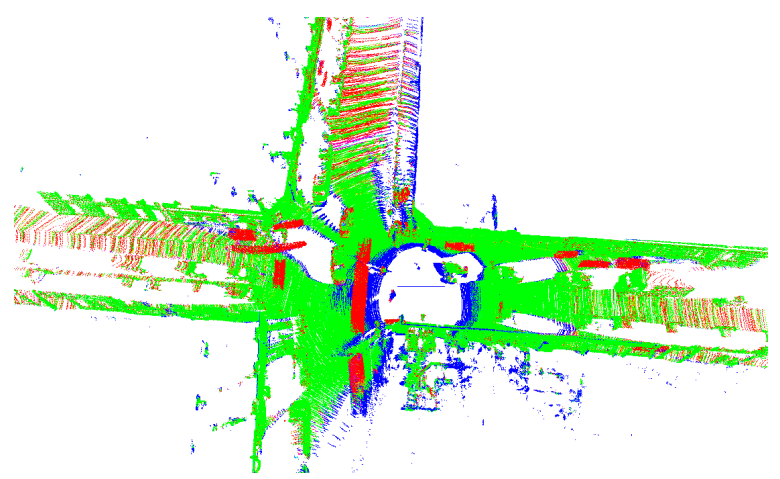

(a)

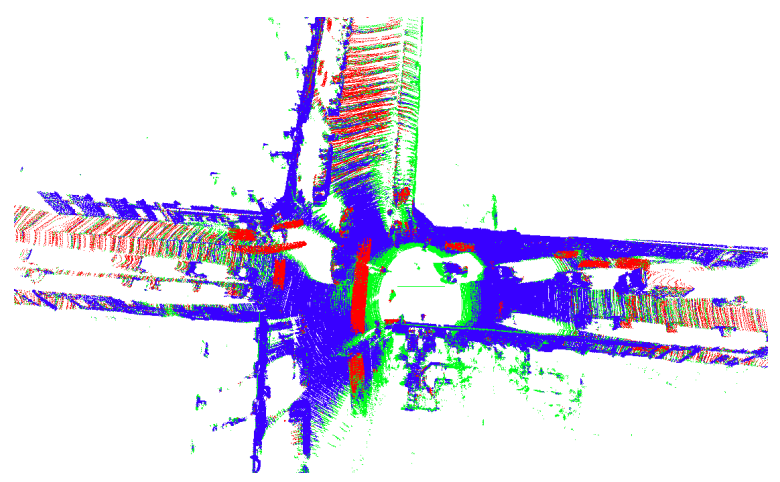

(b)

Figure 8. Moving object detection from a mobile laser scanner.

(a) DST approach (R:moving, G:static, B:uncertain); (b)

Probability approach (R:moving, G:uncertain, B:static)

False alarms are observed on the ground when the points are far from the laser center, in which case the laser beam's incidence angle is large. Given a point on the ground, the laser ray behind will provide a conflicting evidence as the ray is close enough to have an impact as if it passes through the point. Hence the point will be detected as moving. One solution is to incorporate the point normal. The occupancy is then modelled around the local surface defined by the point's normal. For details please refer to Hebel et al. (2013) and Xiao et al. (2015). 
Moving point detection assessment without and with point normals are listed in Table 2. DST approach shows a higher recall and $F_{1}$ in both cases, whereas probability approach gives a better precision. The detection with point normals shows significant improvements for both approaches. The results with normals are illustrated in Figure 9.

\begin{tabular}{|l|l|l|l|l|l|l|}
\hline Method & \multicolumn{3}{|c|}{ No normal } & \multicolumn{3}{c|}{ With normal } \\
\hline \multirow{3}{*}{ DST } & R\% & P\% & $F_{1} \%$ & R\% & P $\%$ & $F_{1} \%$ \\
\cline { 2 - 7 } Probability & $\mathbf{8 1 . 9}$ & 53.9 & $\mathbf{6 5 . 0}$ & $\mathbf{8 0 . 1}$ & 84.2 & $\mathbf{8 2 . 5}$ \\
& 71.1 & $\mathbf{5 7 . 4}$ & 63.6 & 66.0 & $\mathbf{9 2 . 1}$ & 76.9 \\
\hline
\end{tabular}

Table 2. Accuracy assessment of moving object detection at point level from a mobile laser scanner.

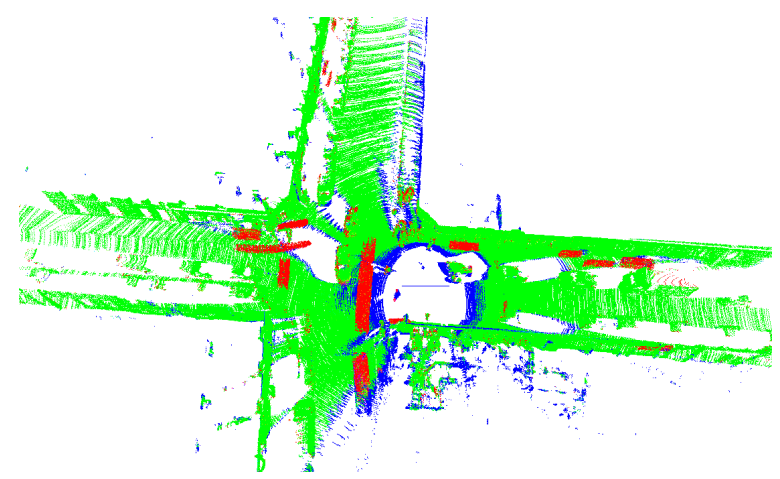

(a)

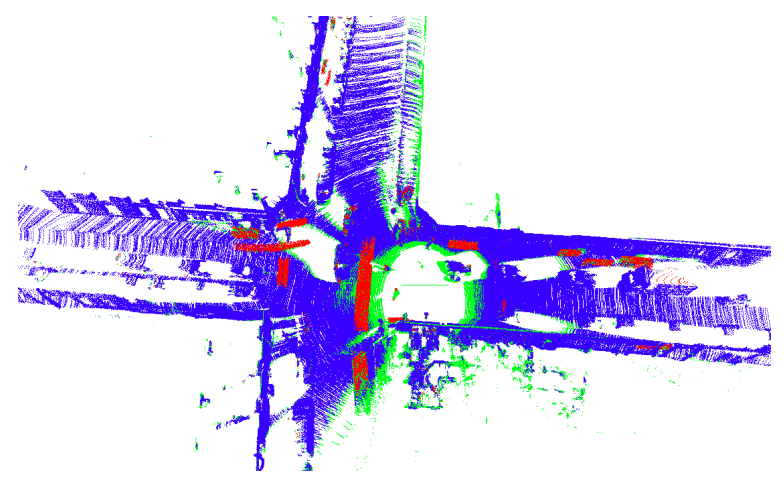

(b)

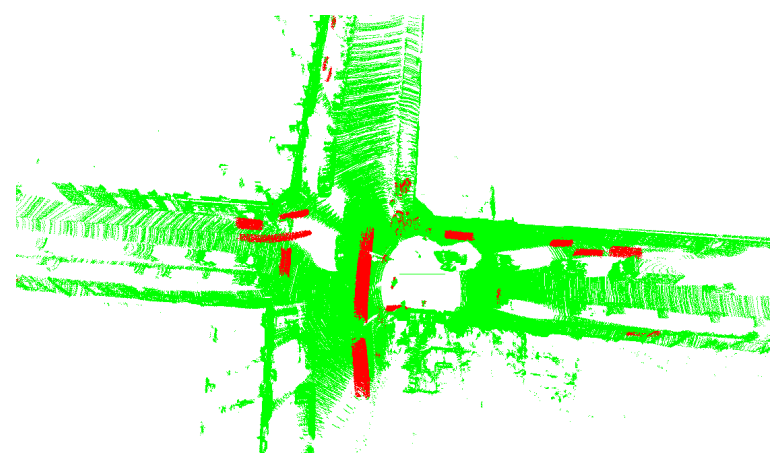

(c)

Figure 9. Moving object detection with point normals using a mobile laser scanner. (a) DST approach (R:moving, G:static, B:uncertain); (b) Probability approach (R:moving, G:uncertain, B:static); (c) ground truth of moving objects (R:moving)

\section{DISCUSSION}

Both DST and probability approaches define the occupancy of space first and fuse the occupancy information from multiple measurements, then efficiently assess the consistency between different scans. The probabilistic occupancy uses only one variable, the probability of being occupied, to model the space. Whereas the DST uses three variables to represent the three different occupancy states, free, occupied and unknown. The advantage is that it is able to distinguish the state of being half free half occupied ( $f=o=0.5)$ and the state of being half occupied half unknown $(o=u=0.5)$. The former happens at the interface between free and occupied slightly before a point. The latter occurs behind the point when occupied $o$ starts to decrease and unknown $u$ to increase. However, for the probability approach, the occupied $P(o)=0.5$ in both cases, meaning there is no differentiation between the case of half free half occupied and the case of unknown. According to Section 2.2.3, the probability approach is actually the same as DST when $u=0$, meaning between the laser center and the point the two methods will have the same results. When $u \in(0,1)$, the unknown evidence will contribute to the occupied value according the definition (Equation 13). In practise, the implementation details may cause difference in terms of uncertainty modelling, occupancy interpolation and simplification, such as log-odds transformation from the probabilistic occupancy.

The logit function is a simplification of the probability where multiplication is transferred to addition. Also to keep the sensitivity to the dynamic scene, the probability is truncated by the clamping thresholds. The the occupancy log-odds values $l_{\text {free }}$ and $l_{\text {occ }}$ have to be carefully chosen. The advantage of probabilistic occupancy is the simplicity and computational efficiency. Note that in the static laser scanning case, the Nearest-point method can be considered as a naive way of interpreting the logodds occupancy method. Each near point is counted as log-odds value 1 , and the clamping threshold is the total number of nearest points. For the DST method, higher weight is given to the consistency evidence to reduce false alarms. However, there is not any mature simplification method such as logit function and clamping. It is anticipated that a similar clamping strategy will also benefit in terms of simplicity and sensitivity to dynamics. Currently the probability approach is preferred for efficient moving object detection. The DST is suitable for precise occupancy modelling and accurate moving object detection. More experiments are needed before drawing a decisive conclusion.

In both approaches, false detections are observed when the ground points are far from the laser center where the incidence angles are large. An effective solution is to model the occupancy along the normal direction instead of the ray direction around the point. Significant improvements are achieved after using the point normals. However, the normals have to be calculated beforehand which may hinder real time or online moving object detection. Majority of these false detections are static ground points, they can either be filtered out first during environment mapping or simply be neglected during moving object tracking as these points will remain still over time. It is a question of the balance between accuracy and efficiency.

\section{CONCLUSION}

Moving object detection is an interesting topic in both robotics and geomatics. This paper has investigated the moving object detection at point level from both static and moving laser scanning 
data using a real-time laser scanner. Two occupancy modelling approaches, DST and Probability, are introduced and compared theoretically. Detailed implementations are explained, from occupancy definition, to evidence fusion, to the final consistency assessment. Similarities and differences are discussed. Both approaches are applied to the two types of laser scanning data. Experimental results are analysed and compared to other moving object detection methods where possible. Further improvement by involving the point normal is also proposed. DST provides a precise framework for occupancy modelling, whereas probability enables proper simplification and sensitivity improvement. Optimised programming will be investigated in the future to further evaluate the computational efficiency of these two approaches.

\section{REFERENCES}

Aijazi, A. K., Checchin, P. and Trassoudaine, L., 2013. Detecting and updating changes in lidar point clouds for automatic $3 \mathrm{~d}$ urban cartography. In: ISPRS Annals of Photogrammetry, Remote Sensing and Spatial Information Sciences, Vol. II-5/W2, pp. 7-12.

Collins, T., 2011. Occupancy grid learning using contextual forward modelling. Journal of Intelligent \& Robotic Systems 64(34), pp. 505-542.

Delafosse, M., Delahoche, L., Clerentin, A., Ricquebourg, V. and Jolly-Desodt, A. M., 2007. A dempster-shafer fusion architecture for the incremental mapping paradigm. In: International Conference on Information Fusion, pp. 1-7.

Dezert, J., Moras, J. and Pannetier, B., 2015. Environment perception using grid occupancy estimation with belief functions. In: International Conference on Information Fusion, pp. 1070-1077.

Girardeau-Montaut, D., Roux, M., Marc, R. and Thibault, G., 2005. Change detection on points cloud data acquired with a ground laser scanner. In: International Archives of the Photogrammetry, Remote Sensing and Spatial Information Sciences, Vol. 36, pp. 30-35.

Grabe, B., Ike, T. and Hoetter, M., 2009. Evidence based evaluation method for grid-based environmental representation. In: International Conference on Information Fusion, pp. 1234-1240.

Hebel, M., Arens, M. and Stilla, U., 2013. Change detection in urban areas by object-based analysis and on-the-fly comparison of multi-view als data. ISPRS Journal of Photogrammetry and Remote Sensing 86, pp. 52-64.

Hornung, A., Wurm, K. M., Bennewitz, M., Stachniss, C. and Burgard, W., 2013. OctoMap: An efficient probabilistic 3D mapping framework based on octrees. Autonomous Robots.

Kaestner, R., Maye, J., Pilat, Y. and Siegwart, R., 2012. Generative object detection and tracking in $3 \mathrm{~d}$ range data. In: IEEE International Conference on Robotics and Automation, pp. 30753081.

Lindenbergh, R. and Pietrzyk, P., 2015. Change detection and deformation analysis using static and mobile laser scanning. Applied Geomatics 7(2), pp. 65-74.

Lindström, M. and Eklundh, J.-O., 2001. Detecting and tracking moving objects from a mobile platform using a laser range scanner. In: IEEE/RSJ International Conference on Intelligent Robots and Systems, Vol. 3, pp. 1364-1369.

Moosmann, F. and Stiller, C., 2011. Velodyne slam. In: IEEE Intelligent Vehicles Symposium, pp. 393-398.
Moosmann, F. and Stiller, C., 2013. Joint self-localization and tracking of generic objects in $3 \mathrm{~d}$ range data. In: IEEE International Conference on Robotics and Automation, pp. 1146-1152.

Moras, J., Dezert, J. and Pannetier, B., 2015. Grid occupancy estimation for environment perception based on belief functions and PCR6. In: SPIE Defense+ Security, p. 94740P.

Murphy, R., 1998. Dempster-shafer theory for sensor fusion in autonomous mobile robots. IEEE Transactions on Robotics and Automation 14(2), pp. 197-206.

Paparoditis, N., Papelard, J., Cannelle, B., Devaux, A., Soheilian, B., David, N. and Houzay, E., 2012. Stereopolis ii: A multi-purpose and multi-sensor 3d mobile mapping system for street visualisation and 3d metrology. Revue Française de Photogrammétrie et de Télédétection 200, pp. 69-79.

Payeur, P., 2003. Fuzzy logic inference for occupancy state modeling and data fusion. In: IEEE International Symposium on Computational Intelligence for Measurement Systems and Applications, pp. 175-180.

Shafer, G., 1992. The dempster-shafer theory. Encyclopedia of artificial intelligence pp. 330-331.

Thrun, S., 2002. Learning occupancy grids with forward sensor models. Autonomous Robots 15, pp. 111-127.

Vallet, B., Xiao, W. and Brédif, M., 2015. Extracting mobile objects in images using a velodyne lidar point cloud. In: ISPRS Annals of the Photogrammetry, Remote Sensing and Spatial Information Sciences, Vol. 1, pp. 247-253.

Vu, T.-D., Burlet, J. and Aycard, O., 2011. Grid-based localization and local mapping with moving object detection and tracking. Information Fusion 12(1), pp. 58-69.

Wolf, D. and Sukhatme, G. S., 2004. Online simultaneous localization and mapping in dynamic environments. In: IEEE International Conference on Robotics and Automation (ICRA), Vol. 2, pp. 1301-1307.

Xiao, W., Vallet, B., Brédif, M. and Paparoditis, N., 2015. Street environment change detection from mobile laser scanning point clouds. ISPRS Journal of Photogrammetry and Remote Sensing 107, pp. 38-49.

Xiao, W., Vallet, B., Schindler, K. and Paparoditis, N., 2016. Simultaneous detection and tracking of pedestrian from panoramic laser scanning data. In: ISPRS Annals of Photogrammetry, Remote Sensing and Spatial Information Sciences, Vol. III-3, pp. 295-302.

Xu, S., Vosselman, G. and Oude Elberink, S., 2013. Detection and classification of changes in buildings from airborne laser scanning data. In: ISPRS Annals of Photogrammetry, Remote Sensing and Spatial Information Sciences, Vol. II-5/W2, pp. 343348 .

Yager, R. R. and Liu, L., 2008. Classic works of the DempsterShafer theory of belief functions. Vol. 219, Springer.

Yguel, M., Aycard, O. and Laugier, C., 2007. Update policy of dense maps: Efficient algorithms and sparse representation. In: International Conference on Field and Service Robotics, Vol. 42, pp. 23-33.

Zhou, L. and Vosselman, G., 2012. Mapping curbstones in airborne and mobile laser scanning data. International Journal of Applied Earth Observation and Geoinformation 18, pp. 293-304. 